\title{
Exercise and inflammation Introduction
}

Rheumatic and musculoskeletal diseases (RMDs) represent a diverse group of noncommunicable diseases that affect primarily the joints, muscles or other tissues of the musculoskeletal system but may also have systemic complications. The European League Against Rheumatism (EULAR) recognizes that there are more than 200 different RMDs, which affect individuals of all ages [1]. Based on a 2011 EULAR Position Paper, the most prevalent RMDs are: osteoarthritis (OA), rheumatoid arthritis (RA), osteoporosis, low back pain, ankylosing spondylitis, psoriatic arthritis, gout, fibromyalgia and connective tissue diseases, such as Systemic Lupus Erythematosus (SLE) and Scleroderma [1]. Collectively, RMDs are characterised by common symptoms - albeit this can vary according to the RMD with the predominant ones being pain, joint damage, functional disability and fatigue. Another major characteristic of many RMDs is inflammation. Inflammation is a normal biological response to infection or tissue damage, which, under normal circumstances, ceases after the clearance of the stimulus. However, inflammatory processes are dysregulated in RMDs and persist in the long-term with significant detrimental consequences in personal and clinical outcomes, and thus, healthcare costs.

\section{A. Inflammatory response and RMDs}

Inflammation is triggered by the innate immune system, once its cells detect infection or tissue injury. This is done by surveillance of pathogens via the involvement of pattern recognition receptors, such as Toll-like receptors, on the cell surface and in the cytoplasm. In autoimmune rheumatic disease, such mechanisms become dysregulated and end up fostering a prolonged activation of chronic inflammatory responses. Nevertheless, developing autoimmune disease is normally prevented in humans via peripheral safety mechanisms that are in place to reduce host tolerance of potentially pathogenic cells [2].

Inflammation can be distinguished in acute and chronic, each characterised by distinct pathways. Acute inflammation involves the local vasculature, the immune system and cells of an injured site. Chronic inflammation, on the other hand, which is what we see in RMDs, is characterized by tissue damage from inflammatory processes, as well as a progressive change in the type of immune cells that are presented in the affected inflamed tissues. There is also a distinction between RMDs with regards to the way that inflammation ensues. For example, RA and SLE are autoimmune RMDs which means that autoantibodies (i.e. antibodies targeting the patient's own proteins) are produced (e.g. rheumatoid factor and anticitrullinated peptide antibodies in RA and anti-nuclear or anti-DNA antibodies in SLE). On the other hand, OA which was traditionally considered a non-inflammatory arthropathy because neutrophils were not present in the synovial fluid, is also now considered to have an early and long-term inflammatory component [3], as denoted by the production of cytokines which are overexpressed in the synovium of OA patients (although at much lower concentrations compared to RA) [4]. It is not within the scope of this review to describe in detail the pathogenic processes involved in each individual RMD, but dysregulation of cytokine production and perpetuation of inflammation is a common characteristic in all RMDs.

Cytokines mediate inflammatory responses in RMDs in many different ways. Proinflammatory cytokines, such as Tumour Necrosis Factor alpha (TNF $\alpha$ ), Interleukins 1 (IL-1) and 6 (IL-6) mediate inflammatory pathways in RMDs, often acting in a synergistic manner. Their kinetics/expression seem to be involved in a vicious cycle where one may trigger an overexpression of the others. The pleiotropic effects of cytokines are not only involved in the pathogenesis of articular damage in RMDs but they also may explain several of their systemic manifestations and comorbidities. 
Pharmacological interventions are the mainstay treatment of inflammation in RMDs but despite significant advances in drug availability and therapeutic strategies in recent decades, there remains a significant treatment deficit. Other interventions with antiinflammatory potential are, therefore, of importance: a major one is increased physical activity. Physical activity is any bodily movement that increases energy expenditure above resting levels, such as walking and doing household activities. Exercise is part of physical activity and is a planned and structured behaviour, such as swimming or exercising in the gym three days a week at specific intensities, that aims to improve specific outcomes, such as functional ability or cardiorespiratory fitness. In the last two decades, a significant amount of work has been conducted to better understand the effects of exercise on inflammatory processes both in the healthy and populations with non-communicable disease. The potential benefits of exercise are recognized and included in recent EULAR projects and recommendations focusing on implementation of physical activity and exercise as "adjuvants" to improve disease-related symptoms and comorbidities in RMDs [5-8]. The present review focuses on the contribution of inflammatory mediators (with a special focus on cytokines as the overarching factor) on disease-related outcomes and systemic manifestations in RMDs, and discusses how exercise dosage, in particular exercise intensity, can mitigate the effects of inflammatory mediators on different outcomes.

\section{B. Exercise and inflammation}

Assessing inflammation as a process is difficult due to the multiple cells, mediators and pathways involved. Exercise-induced effects on inflammation have additional complexity, since they depend on resident macrophages, which exert different effects on different processes depending on the tissue they reside. In this review, the effects of exercise on inflammation will, therefore, be discussed in the context of local, tissue-specific responses.

The target of any intervention in RMDs is remission. Remission conventionally includes significant reductions below certain thresholds of some clinical outcomes (e.g. pain) and markers of the acute phase response (usually the erythrocyte sedimentation rate-ESR, a major determinant of which is fibrinogen, or the acute phase reactant, C-reactive proteinCRP). The acute phase response is regulated by specific pro-inflammatory cytokines, predominantly IL-6. When it comes to assessing the effects of exercise on inflammation, the main focus has been on the local and systemic effects of pro-inflammatory cytokines with the majority of studies having been conducted in athletes and/or the general population. No experimental studies exist to date that have investigated the effects of exercise on inflammation in RMDs as a primary outcome, as denoted in two relevant recent metaanalyses in RMDs investigating the effects of physical activity and/or exercise on disease activity and inflammatory markers $[9,10]$.

\section{B.1. Exercise and Cytokines}

Early sports science studies revealed a marked increase in circulating IL-6 in response to prolonged endurance exercise [11]. Prior to 2000, it was hypothesized that this was the result of muscle damage and subsequent inflammation. Indeed, following marathon running, significant increases were detected in IL-6 mRNA in muscle biopsies and subsequent circulating IL-6, which prompted researchers to suggest that prolonged exercise results in damage in muscle fibres, releasing IL-6 in the circulation and triggering an inflammatory response [12]. This hypothesis was supported by other studies, which revealed associations between creatine kinase (a frequent biomarker used to evaluate muscle damage) and serum IL-6 following high-intensity exercise [13]. This was later questioned, since well-controlled non-damaging exercise protocols still revealed a significant elevation in IL-6 [14]. However, the balance of evidence is that muscle damaging (e.g. eccentric) exercise, particularly in 
untrained people, such as patients with RMDs, does cause an inflammatory response. The effects of prolonged high-intensity exercise-induced muscle damage on inflammation are well described, with neutrophils and macrophages infiltrating the damaged muscle tissue up to 48 hours post exercise [15], suggesting that part of the elevation observed in muscular and circulating IL-6 may be due to activated muscle-residing macrophages in the site of injury. At first sight, such evidence may act as a barrier for people with RMDs to engage in exercise, particularly high-intensity exercise. However, this is a normal process that takes place within the human body to induce increases in muscle mass (and strength), which in turn can reduce functional disability and improve fatigue. It is also important to mention that the manifestations of muscle damage, or delayed onset muscle damage (DOMS), manifests with only mild pain which can be minimized if the high-intensity exercise protocol applies progressively the exercise intensity (i.e. starting from lower intensities and building up to high-intensity dosage). The effects of such exercise protocols on physiological adaptations, local and systemic inflammation, symptoms and function in people with RMDs have not been evaluated.

A growing amount of literature now supports that engagement in exercise induces an anti-inflammatory phenotype in the general population, both acutely as well as in the long term (Figure 1), even though this may be preceded by an acute inflammatory load. The effects of acute exercise on inflammation have been mainly investigated in the muscle by assessing muscle-derived cytokines termed "myokines", while the long-term effects of exercise on inflammation are mainly observed in adipose tissue with adipose tissue-derived cytokines termed "adipokines".

\section{B.1.1. Acute exercise and inflammation}

Muscle contraction during exercise is now thought to be the main trigger for overexpression of IL-6. Exercise-induced IL-6 mRNA within the muscle, increases IL-6 in the circulation which then acts as a trigger to activate hepatic glucogenolysis and lipolysis; this occurs because energy within the muscle is quickly depleted with exercise, so this mechanism triggers pathways to provide additional energy to the exercising muscles [16]. However, this overproduction of IL-6 is not thought to induce inflammation. In support of this, IL-6 in RMDs is induced by macrophages and necessitates the presence of TNF $\alpha$, which is activated by the nuclear factor-kB (NF-kB) pathway [15]. In contrast, muscular exercise-induced production of IL- 6 occurs without the presence of $\mathrm{TNF} \alpha$ or NF-kB activation, and is regulated by an interaction between the nuclear factor of activated T-cells (NFAT) and glycogen-p38 mitogen activated protein kinase pathways (MAPK) [17]. These phenomena appear to coincide with other anti-inflammatory events. Specifically, during exercise, soluble TNF receptors, thought to be naturally produced inhibitors of TNF $\alpha$ [18], increase and remain elevated during and post exercise [15]. The same is true for the IL-1 receptor antagonist (that blocks the actions of IL-1), which is also produced during exercise and remains elevated post exercise [15]. At the same time, IL-1b and TNF $\alpha$, which elevate and mediate the prolonged inflammatory responses seen in autoimmunity, remain supressed during a bout of exercise [15]. Furthermore, muscle-resident pro-inflammatory subtype 1 macrophages (M1) decrease and anti-inflammatory subtype 2 (M2) macrophages increase in response to exercise in healthy people [19] with these effects also impacting on the expression of pro-inflammatory cytokines. These observations suggest that exercise may acutely promote an anti-inflammatory environment within the human body. 


\section{B.1.2. Long-term exercise and inflammation}

The long-term effects of exercise on reducing inflammation are thought to be mainly mediated by reductions in the size of adipose tissue. It is well-established that obesity is a low-grade inflammatory state with adipose tissue acting as an endocrine and paracrine organ responsible for stimulating the increase of inflammatory mediators. In support of this, the expression of inflammatory mediators such IL-6 and TNF $\alpha$ deriving from macrophages residing in adipose tissue cells, are overexpressed in overweight and obese compared to normal weight individuals, while inflammatory processes are activated early in the expansion and progression of adipose tissue [20]. On the other hand, evidence suggests that exercise can have a significant effect in reducing the size of adipose tissue cells. The most recent systematic review and meta-analysis on the effects of exercise vs. diet on adiposity included 4,815 individuals from 117 studies and suggested that exercise results in greater loss of adipose tissue compared to hypocaloric diets while weight remained unchanged [21].

B.2. Regulation of inflammation via induction of the peroxisome proliferator-activated receptor $\gamma$ co-activator $1 \alpha(\mathrm{PGC}-1 \alpha)$

PGC1 $\alpha$ is a transcriptional coactivator that regulates genes involved in energy metabolism and is the predominant regulator of mitochondrial biogenesis. PGC1 $\alpha$ elevates rapidly during exercise - via the AMP-activated protein kinase (AMPK) - possibly to regulate metabolic fluxes in the exercising skeletal muscle as ATP reduces and metabolic demands increase with exercise [22]. Following exercise, PGC1 $\alpha$ mRNA returns to pre-exercise baseline levels [23]. Moreover, changes in the musculature with long-term exercise, such as fiber-type switching towards the more oxidative type IIa and type I muscle fibers, associate with an elevation of PGC-1 $\alpha$ [24]. The induction of PGC1 $\alpha$ with exercise may potentially exert anti-inflammatory effects, since loss of muscle PGC- $1 \alpha$ results in a transcriptional induction of genes that are mediating inflammation [25]. Specifically, in in vivo studies that used PGC-1 $\alpha$ knockout animals, an increased expression of TNF $\alpha$ and IL-6 has been observed [26]. In theory, persistence of such events may suggest that more immune cells may be recruited in such environments, thereby, amplifying a pro-inflammatory phenotype. In addition to this, the anti-inflammatory effects of PGC1 $\alpha$ may be reflected in the downregulation of oxidative stress mediating gene expression in vascular endothelial cells [27]. Finally, PGC1 $\alpha-$ dependent effects are seen within exercising muscles with regards to the polarization from M1 pro-inflammatory to M2 anti-inflammatory macrophages [28]. It is thought that in vivo muscle-induced PGC1 $\alpha$ may not be sufficient to prevent systemic inflammation, however, it can promote an anti-inflammatory environment via inducing anti-inflammatory and suppressing pro-inflammatory cytokines [29].

The biological plausibility to suggest a mediating effect of exercise on muscle and adipose tissue inflammation is strong but studies are available only in the general population. A systematic review and meta-analysis (in 1250 individuals from 11 studies) in healthy middle aged and older adults (similar to the age ranges observed in rheumatic diseases) suggested that aerobic exercise can reduce circulating IL-6 and TNF $\alpha$ [30]. However, relevant studies in inflammatory disease are currently lacking and/or lack the methodological rigour for valid observations. This has been highlighted in a 2019 systematic review in RA [10] and a 2017 meta-analysis in patients with inflammatory RMDs (8) which suggested that the effects of exercise on inflammation are controversial or small and require further investigation. Clearly, much more work is necessary to understand the regulation of inflammatory and antiinflammatory pathways in response to short and long-term exercise engagement in people with RMDs. 


\section{B.3. Exercise and routine serological Assessments of Inflammation}

The most common serological assessments of the effects of any intervention on inflammation in routine clinical practice, are the CRP and ESR.

\section{B.3.1. Exercise and CRP}

The effects of exercise on CRP has received increased scientific attention over the last decade in non-RMD populations. A 2016 systematic review and meta-analysis of 83 randomized controlled trials involving 3769 participants suggested that exercise performed for more than two weeks can reduce CRP [31]. This was more pronounced when exercise was accompanied by reductions in body mass index and fat mass, however, reductions in CRP were still evident even without weight loss [31]. Another 2019 meta-analysis, targeting the effects of aerobic exercise on CRP in healthy middle-aged and older adults (11 studies involving 1,250 participants) confirmed these results. There is also consistency between the findings seen in the general population and those in some non-communicable diseases. For example, a 2016 meta-analysis of studies involving people with cardiovascular disease (CVD) and healthy controls (data from 43 studies and a total of 3575 participants) revealed that exercise can reduce CRP to a similar extent in the two groups [32]. Similar results were observed in people with type 2 diabetes mellitus (meta-analysis of 14 randomised controlled trials and 824 participants) [33]. In contrast, the aforementioned two available systematic reviews in RA and in inflammatory RMDs [9] did not find statistically or clinically significant effects of exercise on CRP. This is probably due to significant variability in the methodological designs of the studies included but it may also be because the size of such an effect may well be detectable in low-grade inflammation states (such as obesity, CVD or type 2 diabetes) but not in high-grade inflammation conditions, such as the RMDs. In this context, it is interesting that a two-year follow-up study in patients with knee OA (a low-grade inflammation RMD) demonstrated that higher CRP levels were related to lower gains in muscle strength [34].

From the mechanistic perspective, the way exercise may affect CRP levels remains unclear. The link between IL-6 mediated induction of the hepatic acute phase response is an obvious possibility. However, some epidemiological studies suggest that the effects of exercise on CRP levels may be due to confounders such as hypertension, body mass-related variables (waist-to-hip, body mass index), age and smoking with which CRP is also associated [35].

\section{B.3.2. Exercise and Fibrinogen}

Produced by hepatocytes in the liver and then circulating in the blood stream, fibrinogen acts primarily to occlude blood vessels to stop bleeding. It is also an acute phase reactant and may exhibit a multi-directional pro-inflammatory role, including the induction of TNF $\alpha$ and IL-1 [36]. Fibrinogen acts on the affinity of red blood cells to bind with one another and is, therefore, a major determinant of the ESR. Fibrinogen was mainly an interest in studies dating 20 years ago, with longitudinal observational studies showing an average reduction of $0.4 \mathrm{~g} / \mathrm{l}$ with endurance training [37], while recent studies also suggested that lower physical activity associates with higher fibrinogen levels in adults with non-communicable diseases [38]. However, these results are contradicted in a more recent randomized controlled trial where no differences were detected in fibrinogen between adult women who engaged in intermittent brisk walking against controls [39]. Both high physical activity and cardiorespiratory fitness have been associated with lower fibrinogen levels in RA patients [40, 41]. However, in the 2019 systematic review on physical activity and inflammation in RA, an exercise effect on ESR was seen in only 3 of the 14 studies included [10]. Another meta-analysis in inflammatory RMDs (8) suggested that the effects of exercise on ESR do exist but they are small [9]. 
Figure 1. Exercise may promote an anti-inflammatory phenotype in different tissues
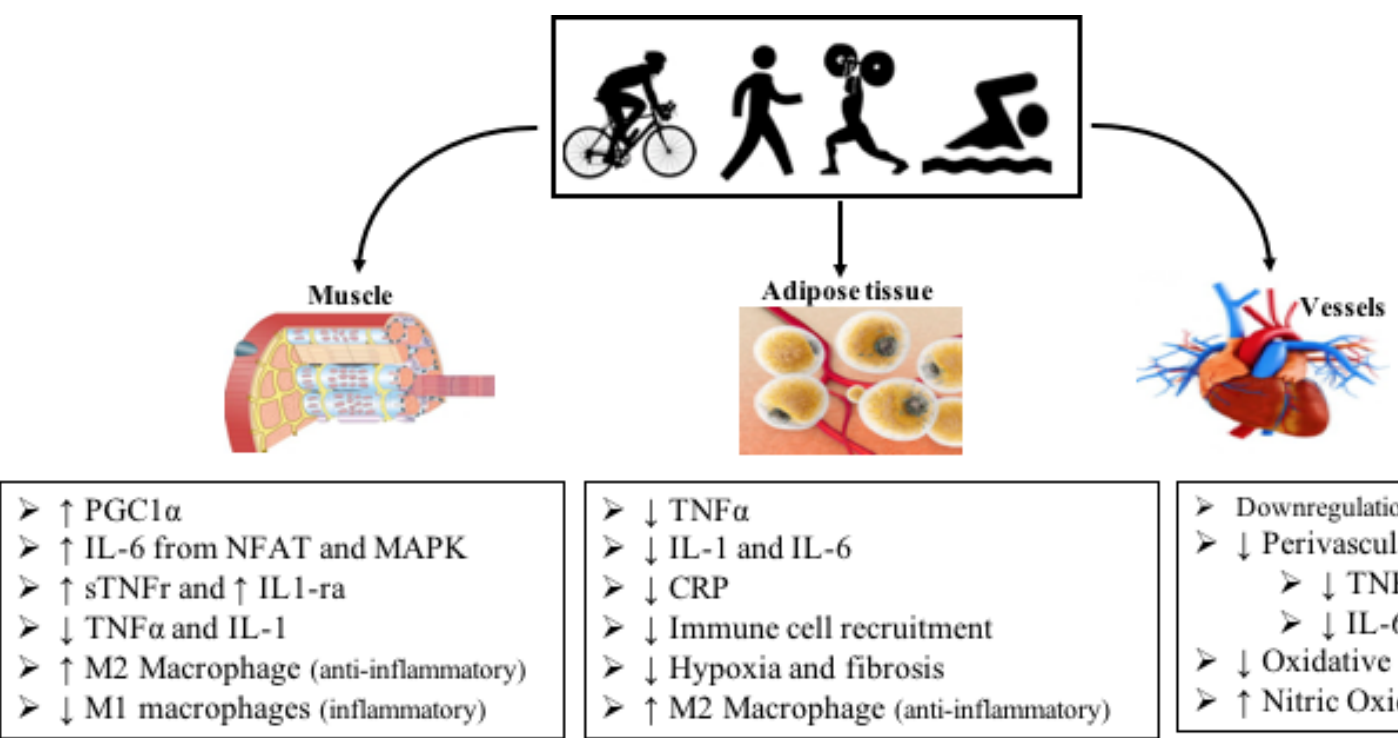

Downregulation of metalloproteinases

$>\downarrow$ Perivascular adipose tissue

$>\downarrow \mathrm{TNF} \alpha$

$>\downarrow$ IL-6

$\downarrow$ Oxidative stress

$>\uparrow$ Nitric Oxide

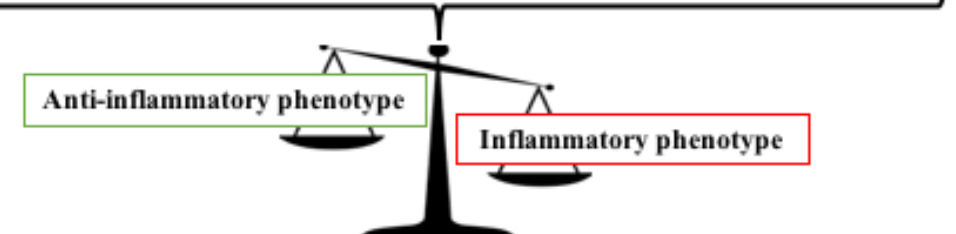

Abbreviations. PGC1 $\alpha$ : peroxisome proliferator-activated receptor $\gamma$ co-activator $1 \alpha$, IL-6: interleukin 6, NFAT: nuclear factor of activated T-cells, MAPK: mitogen activated protein kinase, sTNFr: soluble tumor necrosis factor receptors, IL-1ra: interleukin 1 receptor antagonist, TNF $\alpha$ : tumor necrosis factor alpha, IL-1: interleukin 1, CRP: c-reactive protein

\section{Exercise can blunt inflammatory pathways which can mitigate prevalent systemic manifestations of RMDs}

Compared to the general population, RMDs are characterised by a higher prevalence of systemic manifestations / comorbidities, which have inflammation as a common denominator. These include loss of muscle mass, atherosclerotic CVD, insulin resistance, osteoporosis, depression, propensity to infections, amongst others. The presence of such manifestations may, in turn, affect many significant outcomes, from falls and fractures to quality of life, physical function, family and social engagement, even premature death. Several animal and human studies (almost exclusively in people without RMDs) suggest that through moderating inflammatory pathways, exercise can have a beneficial impact on most of these systemic RMD manifestations. It is beyond the scope of this review to discuss each of these RMD manifestations separately: we are using two of the most prevalent and those where inflammation plays a critical role in their development and progression: loss of muscle mass and atherosclerotic CVD.

C.1. Exercise, inflammation and loss of muscle mass

In inflamamtory diseases: Loss of muscle mass has been noted in early studies of people suffering from rheumatic disease, with Roubenoff and colleagues observing that muscle wasting is probably due to cytokine-driven hypermetabolism in people with RA [42]. However, increased muscle mass loss has been observed in other rheumatic diseases, such as psoriatic arthritis [43] and SLE [44]. There has been increasing research interest to 
understand how muscle mass loss occurs in rheumatic conditions, with most of the research conducted in RA. The current state-of-the-art is that enhanced muscle mass loss in rheumatic disease seems to be due to three different factors. The first is the observed overexpression of cytokines that may lead to hypermetabolism and enhanced loss of muscle mass [42, 45, 46]. In inflammatory states, enhanced muscle proteinolysis seems to occur through the ubiquitinproteasome pathway where $\mathrm{TNF} \alpha$ can stimulate muscle catabolism via an NF-kB process that increases ubiquitin conjugation to muscle proteins, resulting in protein degradation $[47,48]$. Moreover, increased $\mathrm{TNF} \alpha$ significantly affects repair processes of the muscle via the activation of the proteasome system and transcription factor NF-kB leading to decreased expression of MyoD, which is important for replenishing wasted muscle [49]. Secondly, physical inactivity and thus, lack of anabolic muscle stimuli can result in reduced muscle mass [50]. Thirdly, even a single injection of high-dose intramuscular corticosteroids in RA significantly reduces muscle mass with persistent effects [51]. These observations are substantiated in a recent systematic review and meta-analysis suggesting that at least $1 / 3$ of people with RA have significant loss of muscle mass and subsequent increase in fat mass, a condition termed "rheumatoid cachexia" [42]. Given that one of the main mediators of muscle mass loss in rheumatic disease is TNFa, more recent studies focused on the effects of anti-TNF $\alpha$ medication on muscle mass of RMD patients. Interestingly, these studies suggested that blocking TNF $\alpha$ in RA patients starting anti-TNF $\alpha$ biologics, did not reverse the loss in muscle mass $[52,53]$. In contrast, a controlled trial in RA revealed that unlike anti$\mathrm{TNF} \alpha$ medication, high-intensity resistance exercise effectively increased muscle mass and reduce adiposity in RA patients compared to controls doing range of motion exercises only [54]. Unfortunately, this study did not evaluate inflammatory mediators and their effects on muscle mass, so this remains an area for further investigation.

In non-inflammatory musculoskeletal disease: In some RMDs such as fibromyalgia, no changes in body composition have been observed compared to the general population [55]. However, in other RMDs this is not the case. A narrative review suggests that OA is a non-rheumatic disease characterized by significant loss in muscle mass [56]. Inflammation in the vastus lateralis in people with moderate knee OA has been implicated in the loss of muscle mass of OA patients compared with controls and associated with significantly higher IL-6 as well as inflammatory kinases such as abundance of p65 NF- $\kappa \mathrm{B}$, signal transduced and activator of transcription 3 c-Jun N-terminal kinase [57]. How the presence of these inflammatory mediators impacts on reducing muscle mass loss in OA, is still to be elucidated.

\section{C.2. Exercise, inflammation and atherosclerosis}

Exercise can blunt the actions of inflammatory mediators and thus, halt the induction of biological pathways that lead to the development and progression of atherosclerosis [58]. It is well-established that inflammation is involved in the development and progression of atherosclerosis [59]. Oxidized LDL is recognized by local tissue resident macrophages which take it up via their scavenger receptors in an inflammatory process characterized by the activation of the NF-kB and the inflammatory cytokine genes TNF $\alpha$ and IL-1 [60], and form foam cells. The continued stimulation of $\mathrm{TNF} \alpha$ from vascular macrophages in these atherosclerotic/inflammatory lesions recruits further monocytes to the site of vascular injury, resulting in a vicious cycle of inflammatory perseverance and progression of atherosclerosis. Even in advanced atherosclerotic plaques, macrophages are the major players in the continuing inflammatory responses via secreting pro-inflammatory cytokines [61].

Exercise can blunt the processes of atherosclerosis by: a) affecting different macrophage functions and downstream effects on atherosclerotic plaques but also b) reducing oxidized LDL and thus, its associated inflammatory responses. Evidence for this derives both 
from mouse models and human studies. Firstly, activated macrophages in inflamed atherosclerotic plaques produce matrix metalloproteinases which in turn, lead to a disruption of the atherosclerotic plaque and induce thrombosis [59]. Atherosclerotic plaque macrophage-derived metalloproteinases 3 and 8 are downregulated in aerobically exercising mice while at the same time, an overexpression of the inhibitor of metalloproteinase 2 has been observed [62]. As previously mentioned, macrophage-derived pro-inflammatory cytokines from human adipose tissue are also downregulated with exercise [15]. This may be particularly important as increased fat deposition in adjunct coronary tissues may further induce an inflammatory phenotype. Indeed, obese individuals are characterized by increased cardiac adiposity i.e. increased epicardial fat and fatty infiltration in the myocardium. This phenomenon manifests with increased thickness or volume of adipose tissue layers that surround the heart and vessels which promotes myocardial inflammation and dysfunction [63]. Perivascular adipose tissue can secrete TNF $\alpha$ and IL-6, a mechanism that contributes to the ensuing presence of vascular inflammation and oxidative stress, disruption of vascular tone as well as intimal hyperplasia and formation, all leading to the progression of atherosclerosis [64]. In patients undergoing abdominal aorta surgery, it has been observed that perivascular aortic adipose tissue can secrete inflammatory cytokines [65], while TNF $\alpha$ and IL-6 are overexpressed in epicardial adiposity [66]. Potentially the effects of exercise to reduce inflammation may expand on such tissues, thereby blunting inflammatory responses (Figure 1). Indeed, in the absence of weight loss, exercise can reduce perivascular adipose tissue; it can also blunt inflammatory responses from this tissue in obese people engaging in strength and aerobic exercise [67]. With regards to oxidized LDL, trials on healthy and obese adults demonstrate that exercise can have dual effects on the oxidation of LDL and its subsequent regulation of inflammatory genes. These are that exercise can: a) induce resistance to oxidation by improving the body's anti-oxidant capacity while at the same time b) reduce circulating oxidized LDL $[68,69]$, although the exact mechanisms are still unclear. Research in this field in RMDs is currently completely absent.

\section{Dosage of exercise and induction of anti-inflammatory responses}

The exact dosage of exercise that can improve health outcomes, including reducing inflammation, is an ongoing area of investigation. It is exercise rather than habitual physical activity that has been the main focus with regards to inflammatory responses, since exercise seems to have a dose-dependent relationship with health outcomes and inflammatory mediators. Nevertheless, new emerging areas, such as sedentary behaviours and lightintensity activity and their impact on inflammation is a rapidly developing field in healthcare sciences including RMDs [70], particularly due to the observed positive associations between both of these with vascular dysfunction and cardiovascular risk [71].

Specific public health recommendations are available for physical activity engagement. When individuals engage in 150-300 $\mathrm{min} /$ week of moderate or 75-150 min/week of strenuous physical activity coupled with resistance training twice a week, multiple different benefits on health can ensue. For exercise, on the other hand, the American College of Sports Medicine (ACSM) has conducted substantial work in defining the beneficial exercise dosage, using the frequency, intensity, time and type (FITT) of exercise as determinants of dosage (Table 1) [72]. 
Table 1. Required dosage of exercise for health benefits from the ACSM and in RMDs

\begin{tabular}{|l|l|l|}
\hline & Aerobic Exercise & Resistance Exercises \\
\hline Intensity & $55-90 \%$ of HRmax & $55-90 \%$ of HRmax / 60-85\% of 1 RM \\
\hline Duration & $20-90$ min & $\begin{array}{l}\text { 8-12 repetitions, 2-4 sets. Exercises should induce } \\
\text { fatigue but not exhaustion }\end{array}$ \\
\hline Frequency & 3-5 days / week & 2-3 days / week \\
\hline Abbreviations: HRmax: maximum heart rate, RM: repetition maximum \\
\hline
\end{tabular}

It is agreed currently that, although a minimum dosage is required to increase cardiorespiratory fitness, the lack of consistent findings in this area is an issue of concern, and predominantly influenced by the initial level of fitness / conditioning of the exercising participants [73]. This is also the case in RMDs. Baseline levels of cardiorespiratory fitness as well as levels of physical activity are low in people with RMDs [7, 41] and in specific RMDs, such as RA, they are alarmingly low [40]. Identifying the dosage of exercise is recognized as an important matter in RMD research, with studies that adopt the ACSM guidelines on exercise dosage, reporting significantly higher benefits in pain of people with OA, compared to those not adhering to the equivalent guidelines [74]. Whether the same stands true for the dosage of exercise on inflammatory responses is not currently known and has not been investigated in RMD research

The exercise dosage from the studies included in the two available systematic reviews on inflammation and exercise in RMDs $[9,10]$ was collectively within the dosage suggested by the ACSM.FITT principle details were missing from the older studies due to lack of exercise dosage recommendations at the time. However, even more recent exercise studies are, unfortunately, characterized by the same issues. Research scientists investigating exercise and health outcomes in RMDs should do much better in reporting the FITT principles of the exercise interventions they conduct in order to understand better the effects of different doses of exercise. The exact same issues are present in other areas of investigation, whereby the FITT principles are also not adequately reported, such as exercise and cancer research [75]. In addition to this, the recent EULAR physical recommendations also do not focus on the dosage of exercise on different health outcomes in RMDs [7].

Within these ongoing limitations, targets for exercise dosage should exist and appropriately tested. These targets should comply with the ACSM exercise dosage (Table 1), given that multiple different benefits are seen in the general population, people with noncommunicable disease [72] as well as RMDs [7, 76-78]. The same is true for inflammation. It is important for exercise programs to consider the baseline levels of functional ability and cardiorespiratory fitness of people with RMDs, as well as their preferences in order to tailor exercise programs, and most importantly, to apply the exercise intensity in a progressive manner, to avoid injury. So, although lower intensities can be applied at the early stages of an exercise intervention, the intensity of the exercise should progressively build up within a few weeks, to reach the ACSM suggested dosage, as an optimal way of improving disease-related outcomes, comorbidities as well as reduce inflammation. 


\section{Practice Points}

- In the general population, there seems to be biological plausibility suggesting that exercise may promote an acute and long-term anti-inflammatory phenotype

- The potential anti-inflammatory effects of exercise may blunt biological pathways that associate with and/or cause comorbidities

- In people with rheumatic and musculoskeletal disease, the anti-inflammatory effects of exercise may help manage inflammation and inflammation-driven comorbidities better, however, research evidence is still at preliminary stages

- The dosage of exercise requires individualization while at the same time it needs to adhere to specific targets (frequency, intensity, time and type) in order to optimize the management of symptoms in rheumatic and musculoskeletal diseases as well as other non-communicable diseases.

\section{Research Agenda}

There is biological plausibility that the benefits of short- and long-term exercise on inflammation seen in the general population and populations with other non-communicable diseases could be extended in rheumatic and musculoskeletal diseases (RMDs), but in the absence of relevant basic, epidemiological and clinical trial evidence specifically in RMDs, firm conclusions cannot be made.

To understand the effects of exercise on inflammation in rheumatic and musculoskeletal diseases, appropriately designed and powered studies should aim to address the following aspects:

- What are the effects of exercise on markers of inflammation (CRP and/or ESR) in people with RMDs?

- What are the associations between different levels, types and intensities of physical activity and/or exercise and inflammatory markers in RMDs?

- How does exercise work in the context of pharmacological therapy, particularly NSAIDs, corticosteroids, conventional and biologic DMARDs?

\section{Conclusion}

In the general population and populations living with non-communicable disease there is evidence to suggest that exercise has anti-inflammatory effects. Relevant studies in RMDs are very few, possibly due to the complexity of studying relevant mechanisms in the context of high-grade inflammatory disorders and their treatments. More targeted research is required in this area of investigation to understand the effects of exercise on inflammation in RMDs. 


\section{References}

1. EULAR. EULAR's position and recomendations. European Lesgue Against Rheumatism. Position paper.

https://www.eular.org/myUploadData/files/EU_Horizon_2020_EULAR_position_paper.pdf. 2011.

2. Hammerling GJ, Schonrich G, Momburg F, Auphan N, Malissen M, Malissen B, et al. Non-deletional mechanisms of peripheral and central tolerance: studies with transgenic mice with tissue-specific expression of a foreign MHC class I antigen. Immunol Rev 1991;122:47-67.

3. Mathiessen A, Conaghan PG. Synovitis in osteoarthritis: current understanding with therapeutic implications. Arthritis Research and Therapy 2019;19(18):DOI 10.1186/s13075017-1229-9.

4. Goldring MB, Otero M. Inflammation in osteoarthritis. Curr Opin Rheumatol 2011;23(5):471-8.

5. Agca R, Heslinga SC, Rollefstad S, Heslinga M, McInnes IB, Peters MJ, et al. EULAR recommendations for cardiovascular disease risk management in patients with rheumatoid arthritis and other forms of inflammatory joint disorders: 2015/2016 update. Ann Rheum Dis 2017;76(1):17-28.

6. Geenen R, Overman CL, Christensen R, Asenlof P, Capela S, Huisinga KL, et al. EULAR recommendations for the health professional's approach to pain management in inflammatory arthritis and osteoarthritis. Ann Rheum Dis 2018;77(6):797-807.

7. Rausch Osthoff AK, Niedermann K, Braun J, Adams J, Brodin N, Dagfinrud H, et al. 2018 EULAR recommendations for physical activity in people with inflammatory arthritis and osteoarthritis. Ann Rheum Dis 2018.

8. Metsios GS, Fenton SAM, Moe HR, van der Esch M, Veldhuijzen van Zanten JJCS, Koutedakis Y, et al. IMplementation of Physical Activity into routine Clinical pracTice in Rheumatic Musculoskeletal Disease: The IMPACT-RMD study protocol and rationale. Mediterr J Rheumatol 2019;30(4):231-6.

9. Sveaas SH, Smedslund G, Hagen KB, Dagfinrud H. Effect of cardiorespiratory and strength exercises on disease activity in patients with inflammatory rheumatic diseases: a systematic review and meta-analysis. Br J Sports Med 2017;51(14):1065-72.

10. Burghardt RD, Kazim MA, Ruther W, Niemeier A, Strahl A. The impact of physical activity on serum levels of inflammatory markers in rheumatoid arthritis: a systematic literature review. Rheumatol Int 2019;39(5):793-804.

11. Nehlsen-Cannarella SL, Fagoaga OR, Nieman DC, Henson DA, Butterworth DE, Schmitt RL, et al. Carbohydrate and the cytokine response to $2.5 \mathrm{~h}$ of running. J Appl Physiol (1985) 1997;82(5):1662-7.

12. Ostrowski K, Rohde T, Zacho M, Asp S, Pedersen BK. Evidence that interleukin-6 is produced in human skeletal muscle during prolonged running. J Physiol 1998;508 ( Pt 3):949-53.

13. Bruunsgaard H, Galbo H, Halkjaer-Kristensen J, Johansen TL, MacLean DA, Pedersen BK. Exercise-induced increase in serum interleukin-6 in humans is related to muscle damage. J Physiol 1997;499 ( Pt 3):833-41.

14. Starkie RL, Arkinstall MJ, Koukoulas I, Hawley JA, Febbraio MA. Carbohydrate ingestion attenuates the increase in plasma interleukin-6, but not skeletal muscle interleukin-6 mRNA, during exercise in humans. J Physiol 2001;533(Pt 2):585-91.

15. Pedersen BK, Febbraio MA. Muscle as an endocrine organ: focus on muscle-derived interleukin-6. Physiol Rev 2008;88(4):1379-406. 
16. Benatti FB, Pedersen BK. Exercise as an anti-inflammatory therapy for rheumatic diseases-myokine regulation. Nat Rev Rheumatol 2015;11(2):86-97.

17. Munoz-Canoves P, Scheele C, Pedersen BK, Serrano AL. Interleukin-6 myokine signaling in skeletal muscle: a double-edged sword? FEBS J 2013;280(17):4131-48.

18. Akira S, Kishimoto T. IL-6 and NF-IL6 in acute-phase response and viral infection. Immunol Rev 1992;127:25-50.

19. Gordon PM, Liu D, Sartor MA, IglayReger HB, Pistilli EE, Gutmann L, et al. Resistance exercise training influences skeletal muscle immune activation: a microarray analysis. J Appl Physiol (1985) 2012;112(3):443-53.

20. Saltiel AR, Olefsky JM. Inflammatory mechanisms linking obesity and metabolic disease. J Clin Invest 2017;127(1):1-4.

21. Verheggen RJ, Maessen MF, Green DJ, Hermus AR, Hopman MT, Thijssen DH. A systematic review and meta-analysis on the effects of exercise training versus hypocaloric diet: distinct effects on body weight and visceral adipose tissue. Obes Rev 2016;17(8):66490 .

22. Hood DA, Irrcher I, Ljubicic V, Joseph AM. Coordination of metabolic plasticity in skeletal muscle. J Exp Biol 2006;209(Pt 12):2265-75.

23. Pilegaard H, Saltin B, Neufer PD. Exercise induces transient transcriptional activation of the PGC-1alpha gene in human skeletal muscle. J Physiol 2003;546(Pt 3):851-8.

24. Russell AP, Feilchenfeldt J, Schreiber S, Praz M, Crettenand A, Gobelet C, et al. Endurance training in humans leads to fiber type-specific increases in levels of peroxisome proliferator-activated receptor-gamma coactivator-1 and peroxisome proliferator-activated receptor-alpha in skeletal muscle. Diabetes 2003;52(12):2874-81.

25. Handschin C, Chin S, Li P, Liu F, Maratos-Flier E, Lebrasseur NK, et al. Skeletal muscle fiber-type switching, exercise intolerance, and myopathy in PGC-1alpha musclespecific knock-out animals. J Biol Chem 2007;282(41):30014-21.

26. Handschin C, Choi CS, Chin S, Kim S, Kawamori D, Kurpad AJ, et al. Abnormal glucose homeostasis in skeletal muscle-specific PGC-1alpha knockout mice reveals skeletal muscle-pancreatic beta cell crosstalk. J Clin Invest 2007;117(11):3463-74.

27. Valle I, Alvarez-Barrientos A, Arza E, Lamas S, Monsalve M. PGC-1alpha regulates the mitochondrial antioxidant defense system in vascular endothelial cells. Cardiovasc Res 2005;66(3):562-73.

28. Dinulovic I, Furrer R, Di Fulvio S, Ferry A, Beer M, Handschin C. PGC-1alpha modulates necrosis, inflammatory response, and fibrotic tissue formation in injured skeletal muscle. Skelet Muscle 2016;6:38.

29. Eisele PS, Furrer R, Beer M, Handschin C. The PGC-1 coactivators promote an antiinflammatory environment in skeletal muscle in vivo. Biochem Biophys Res Commun 2015;464(3):692-7.

30. Zheng G, Qiu P, Xia R, Lin H, Ye B, Tao J, et al. Effect of Aerobic Exercise on Inflammatory Markers in Healthy Middle-Aged and Older Adults: A Systematic Review and Meta-Analysis of Randomized Controlled Trials. Front Aging Neurosci 2019;11:98.

31. Fedewa MV, Hathaway ED, Ward-Ritacco CL. Effect of exercise training on C reactive protein: a systematic review and meta-analysis of randomised and non-randomised controlled trials. Br J Sports Med 2017;51(8):670-6.

32. Hammonds TL, Gathright EC, Goldstein CM, Penn MS, Hughes JW. Effects of exercise on c-reactive protein in healthy patients and in patients with heart disease: A metaanalysis. Heart Lung 2016;45(3):273-82.

33. Hayashino Y, Jackson JL, Hirata T, Fukumori N, Nakamura F, Fukuhara S, et al. Effects of exercise on C-reactive protein, inflammatory cytokine and adipokine in patients 
with type 2 diabetes: a meta-analysis of randomized controlled trials. Metabolism 2014;63(3):431-40.

34. Sanchez-Ramirez DC, van der Leeden M, van der Esch M, Roorda LD, Verschueren $\mathrm{S}$, van Dieen JH, et al. Elevated C-reactive protein is associated with lower increase in knee muscle strength in patients with knee osteoarthritis: a 2-year follow-up study in the Amsterdam Osteoarthritis (AMS-OA) cohort. Arthritis Res Ther 2014;16(3):R123.

35. Kasapis C, Thompson PD. The effects of physical activity on serum C-reactive protein and inflammatory markers: a systematic review. J Am Coll Cardiol 2005;45(10):1563-9.

36. Davalos D, Akassoglou K. Fibrinogen as a key regulator of inflammation in disease. Semin Immunopathol 2012;34(1):43-62.

37. Ernst E. Regular exercise reduces fibrinogen levels: a review of longitudinal studies. Br J Sports Med 1993;27(3):175-6.

38. Gomez-Marcos MA, Recio-Rodriguez JI, Patino-Alonso MC, Martinez-Vizcaino V, Martin-Borras C, de-la-Cal-Dela-Fuente A, et al. Relationship between physical activity and plasma fibrinogen concentrations in adults without chronic diseases. PLoS One 2014;9(2):e87954.

39. Furukawa F, Kazuma K, Kojima M, Kusukawa R. Effects of an off-site walking program on fibrinogen and exercise energy expenditure in women. Asian Nurs Res (Korean Soc Nurs Sci) 2008;2(1):35-45.

40. Metsios GS, Koutedakis Y, Veldhuijzen van Zanten JJ, Stavropoulos-Kalinoglou A, Vitalis P, Duda JL, et al. Cardiorespiratory fitness levels and their association with cardiovascular profile in patients with rheumatoid arthritis: a cross-sectional study. Rheumatology (Oxford) 2015;54(12):2215-20.

41. Metsios GS, Stavropoulos-Kalinoglou A, Panoulas VF, Wilson M, Nevill AM, Koutedakis Y, et al. Association of physical inactivity with increased cardiovascular risk in patients with rheumatoid arthritis. Eur J Cardiovasc Prev Rehabil 2009;16(2):188-94. 42. Roubenoff R, Roubenoff RA, Cannon JG, Kehayias JJ, Zhuang H, Dawson-Hughes $\mathrm{B}$, et al. Rheumatoid cachexia: cytokine-driven hypermetabolism accompanying reduced body cell mass in chronic inflammation. J Clin Invest 1994;93(6):2379-86.

43. Krajewska-Wlodarczyk M, Owczarczyk-Saczonek A, Placek W. Changes in body composition and bone mineral density in postmenopausal women with psoriatic arthritis. Reumatologia 2017;55(5):215-21.

44. Kipen Y, Briganti EM, Strauss BJ, Littlejohn GO, Morand EF. Three year follow-up of body composition changes in pre-menopausal women with systemic lupus erythematosus. Rheumatology (Oxford) 1999;38(1):59-65.

45. Metsios GS, Stavropoulos-Kalinoglou A, Panoulas VF, Koutedakis Y, Nevill AM, Douglas KM, et al. New resting energy expenditure prediction equations for patients with rheumatoid arthritis. Rheumatology (Oxford) 2008;47(4):500-6.

46. Stavropoulos-Kalinoglou A, Metsios GS, Koutedakis Y, Nevill AM, Douglas KM, Jamurtas A, et al. Redefining overweight and obesity in rheumatoid arthritis patients. Ann Rheum Dis 2007;66(10):1316-21.

47. Lecker SH, Solomon V, Mitch WE, Goldberg AL. Muscle protein breakdown and the critical role of the ubiquitin-proteasome pathway in normal and disease states. J Nutr 1999;129(1S Suppl):227S-37S.

48. Gould DW, Lahart I, Carmichael AR, Koutedakis Y, Metsios GS. Cancer cachexia prevention via physical exercise: molecular mechanisms. J Cachexia Sarcopenia Muscle 2013;4(2):111-24. 
49. Guttridge DC, Mayo MW, Madrid LV, Wang CY, Baldwin AS, Jr. NF-kappaBinduced loss of MyoD messenger RNA: possible role in muscle decay and cachexia. $\underline{\text { Science }}$ 2000;289(5488):2363-6.

50. Summers GD, Metsios GS, Stavropoulos-Kalinoglou A, Kitas GD. Rheumatoid cachexia and cardiovascular disease. Nat Rev Rheumatol 2010;6(8):445-51.

51. Lemmey AB, Wilkinson TJ, Perkins CM, Nixon LA, Sheikh F, Jones JG, et al. Muscle loss following a single high-dose intramuscular injection of corticosteroids to treat disease flare in patients with rheumatoid arthritis. Eur J Rheumatol 2018;5(3):160-4.

52. Marcora SM, Chester KR, Mittal G, Lemmey AB, Maddison PJ. Randomized phase 2 trial of anti-tumor necrosis factor therapy for cachexia in patients with early rheumatoid arthritis. Am J Clin Nutr 2006;84(6):1463-72.

53. Metsios GS, Stavropoulos-Kalinoglou A, Douglas KM, Koutedakis Y, Nevill AM, Panoulas VF, et al. Blockade of tumour necrosis factor-alpha in rheumatoid arthritis: effects on components of rheumatoid cachexia. Rheumatology (Oxford) 2007;46(12):1824-7.

54. Lemmey AB, Marcora SM, Chester K, Wilson S, Casanova F, Maddison PJ. Effects of high-intensity resistance training in patients with rheumatoid arthritis: a randomized controlled trial. Arthritis Rheum 2009;61(12):1726-34.

55. Segura-Jimenez V, Aparicio VA, Alvarez-Gallardo IC, Carbonell-Baeza A, TorneroQuinones I, Delgado-Fernandez M. Does body composition differ between fibromyalgia patients and controls? the al-Andalus project. Clin Exp Rheumatol 2015;33(1 Suppl 88):S2532.

56. Shorter E, Sannicandro AJ, Poulet B, Goljanek-Whysall K. Skeletal Muscle Wasting and Its Relationship With Osteoarthritis: a Mini-Review of Mechanisms and Current Interventions. Curr Rheumatol Rep 2019;21(8):40.

57. Noehren B, Kosmac K, Walton RG, Murach KA, Lyles MF, Loeser RF, et al. Alterations in quadriceps muscle cellular and molecular properties in adults with moderate knee osteoarthritis. Osteoarthritis Cartilage 2018;26(10):1359-68.

58. Metsios GS, Moe RH, van der Esch M, van Zanten J, Fenton SAM, Koutedakis Y, et al. The effects of exercise on cardiovascular disease risk factors and cardiovascular physiology in rheumatoid arthritis. Rheumatol Int 2019; in press. doi: 10.1007/s00296-01904483-6.

59. Libby P. Inflammation in atherosclerosis. Nature 2002;420(6917):868-74.

60. Jovinge S, Ares MP, Kallin B, Nilsson J. Human monocytes/macrophages release TNF-alpha in response to Ox-LDL. Arterioscler Thromb Vasc Biol 1996;16(12):1573-9.

61. Moore KJ, Sheedy FJ, Fisher EA. Macrophages in atherosclerosis: a dynamic balance. Nat Rev Immunol 2013;13(10):709-21.

62. Kadoglou NP, Moustardas P, Kapelouzou A, Katsimpoulas M, Giagini A, Dede E, et al. The anti-inflammatory effects of exercise training promote atherosclerotic plaque stabilization in apolipoprotein E knockout mice with diabetic atherosclerosis. Eur J Histochem 2013;57(1):e3.

63. Guzzardi MA, Iozzo P. Fatty heart, cardiac damage, and inflammation. Rev Diabet Stud 2011;8(3):403-17.

64. Wang D, Wang Z, Zhang L, Wang Y. Roles of Cells from the Arterial Vessel Wall in Atherosclerosis. Mediators Inflamm 2017;2017:8135934.

65. Kranendonk ME, van Herwaarden JA, Stupkova T, de Jager W, Vink A, Moll FL, et al. Inflammatory characteristics of distinct abdominal adipose tissue depots relate differently to metabolic risk factors for cardiovascular disease: distinct fat depots and vascular risk factors. Atherosclerosis 2015;239(2):419-27. 
66. Hirata Y, Kurobe H, Akaike M, Chikugo F, Hori T, Bando Y, et al. Enhanced inflammation in epicardial fat in patients with coronary artery disease. Int Heart J 2011;52(3):139-42.

67. Lee S, Norheim F, Langleite TM, Noreng HJ, Storas TH, Afman LA, et al. Effect of energy restriction and physical exercise intervention on phenotypic flexibility as examined by transcriptomics analyses of mRNA from adipose tissue and whole body magnetic resonance imaging. Physiol Rep 2016;4(21).

68. Elosua R, Molina L, Fito M, Arquer A, Sanchez-Quesada JL, Covas MI, et al. Response of oxidative stress biomarkers to a 16-week aerobic physical activity program, and to acute physical activity, in healthy young men and women. Atherosclerosis

2003;167(2):327-34.

69. Park JH, Park H, Lim ST, Park JK. Effects of a 12-week healthy-life exercise program on oxidized low-density lipoprotein cholesterol and carotid intima-media thickness in obese elderly women. J Phys Ther Sci 2015;27(5):1435-9.

70. Fenton SAM, Veldhuijzen van Zanten J, Duda JL, Metsios GS, Kitas GD. Sedentary behaviour in rheumatoid arthritis: definition, measurement and implications for health. Rheumatology (Oxford) 2018;57(2):213-26.

71. Fenton SAM, Sandoo A, Metsios GS, Duda JL, Kitas GD, Veldhuijzen van Zanten J. Sitting time is negatively related to microvascular endothelium-dependent function in rheumatoid arthritis. Microvasc Res 2018;117:57-60.

72. Garber CE, Blissmer B, Deschenes MR, Franklin BA, Lamonte MJ, Lee IM, et al. American College of Sports Medicine position stand. Quantity and quality of exercise for developing and maintaining cardiorespiratory, musculoskeletal, and neuromotor fitness in apparently healthy adults: guidance for prescribing exercise. Med Sci Sports Exerc 2011;43(7):1334-59.

73. Swain DP, Franklin BA. VO(2) reserve and the minimal intensity for improving cardiorespiratory fitness. Med Sci Sports Exerc 2002;34(1):152-7.

74. Bartholdy C, Juhl C, Christensen R, Lund H, Zhang W, Henriksen M. The role of muscle strengthening in exercise therapy for knee osteoarthritis: A systematic review and meta-regression analysis of randomized trials. Semin Arthritis Rheum 2017;47(1):9-21.

75. Neil-Sztramko SE, Winters-Stone KM, Bland KA, Campbell KL. Updated systematic review of exercise studies in breast cancer survivors: attention to the principles of exercise training. Br J Sports Med 2019;53(8):504-12.

76. Hurkmans E, van der Giesen FJ, Vliet Vlieland TP, Schoones J, Van den Ende EC. Dynamic exercise programs (aerobic capacity and/or muscle strength training) in patients with rheumatoid arthritis. Cochrane Database Syst Rev 2009(4):CD006853.

77. Metsios GS, Stavropoulos-Kalinoglou A, Veldhuijzen van Zanten JJ, Treharne GJ, Panoulas VF, Douglas KM, et al. Rheumatoid arthritis, cardiovascular disease and physical exercise: a systematic review. Rheumatology (Oxford) 2008;47(3):239-48.

78. Rongen-van Dartel SA, Repping-Wuts H, Flendrie M, Bleijenberg G, Metsios GS, van den Hout WB, et al. Effect of Aerobic Exercise Training on Fatigue in Rheumatoid Arthritis: A Meta-Analysis. Arthritis Care Res (Hoboken) 2015;67(8):1054-62. 